\title{
Single-Arm, Open-Label, Multicenter Study to Evaluate the Safety and Performance of Dura Sealant Patch in Reducing Cerebrospinal Fluid Leakage Following Elective Cranial Surgery: The ENCASE Trial Study Protocol
}

van Doormaal, Tristan P C ; Germans, Menno R ; Sie, Mariska ; Brouwers, Bart ; Fierstra, Jorn ; Depauw, Paul R A M ; Robe, Pierre A ; Regli, Luca

\begin{abstract}
BACKGROUND: Cerebrospinal fluid (CSF) leakage is one of the most common neurosurgical complications, occurring in $4 \%$ to $32 \%$ of surgical cases, with a higher incidence in complicated skull base surgery, intradural spine surgery, and the surgery of the posterior fossa. Our group developed a Dural Sealant Patch (DSP) for watertight dural closure after cranial surgery. OBJECTIVE: To clinically study for the first time the safety and performance of the DSP as a means of reducing CSF leakage in patients undergoing elective cranial intradural surgery with a dural closure procedure. METHODS: We will conduct an open-label, single-arm, multicenter study with a $360 \mathrm{~d}$ (12 mo) follow-up. A total of 40 patients will be enrolled at 3 sites. The primary endpoint is a combination of occurrences of one of the following events: postoperative percutaneous CSF leakage, intraoperative leakage at $20 \mathrm{~cm} \mathrm{H} 2 \mathrm{O}$, or postoperative wound infection. The secondary endpoints are pseudomeningocele and thickness of dura + DSP. EXPECTED OUTCOMES: Not more than 3 patients will meet the primary endpoint suggesting safety and efficacy. DISCUSSION: As a next step, a randomized controlled trial against the best current practice will follow to evaluate if DSP reduces CSF leakage while its safety is noninferior. (C) Congress of Neurological Surgeons 2019.
\end{abstract}

DOI: https://doi.org/10.1093/neuros/nyz396

Posted at the Zurich Open Repository and Archive, University of Zurich ZORA URL: https://doi.org/10.5167/uzh-176961

Journal Article

Published Version

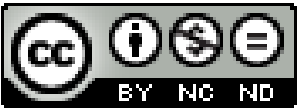

The following work is licensed under a Creative Commons: Attribution-NonCommercial-NoDerivatives 4.0 International (CC BY-NC-ND 4.0) License.

Originally published at:

van Doormaal, Tristan P C; Germans, Menno R; Sie, Mariska; Brouwers, Bart; Fierstra, Jorn; Depauw, Paul R A M; Robe, Pierre A; Regli, Luca (2020). Single-Arm, Open-Label, Multicenter Study to Evaluate the Safety and Performance of Dura Sealant Patch in Reducing Cerebrospinal Fluid Leakage Following Elective Cranial Surgery: The ENCASE Trial Study Protocol. Neurosurgery, 86(2):E203-E208.

DOI: https://doi.org/10.1093/neuros/nyz396 
Tristan P. C. van Doormaal, MD, PhD $\odot * \sharp \S \uparrow$

Menno R. Germans, MD, $\mathrm{PhD}^{\S \uparrow}$

Mariska Sie, MD, PhD\|

Bart Brouwers, MD, PhD* Jorn Fierstra, MD, PhD $\$$

Paul R. A. M. Depauw, MD, PhDll

Pierre A. Robe, MD, PhD* Luca Regli, MD

*Department of Neurosurgery, Brain Center Rudolph Magnus, University Medical Centre Utrecht, Utrecht, The Netherlands; ${ }^{\ddagger}$ Brain Technology Institute, Utrecht, The Netherlands; ${ }^{\S}$ Department of Neurosurgery, University Hospital Zurich, Zurich, Switzerland; "Clinical Neuroscience Center Zurich, Zurich, Switzerland; "Department of Neurosurgery, Elisabeth-TweeSteden Hospital, Tilburg, The Netherlands

\section{Correspondence:}

Tristan P.C. van Doormaal, MD, PhD, University Medical Centre Utrecht, Department of Neurosurgery, G.03.124, Heidelberglaan 100 ,

3584 CX Utrecht, The Netherlands. Email: T.p.c.vandoormaal@umcutrecht.nl

Received, April 25, 2019.

Accepted, July 24, 2019.

(C) Congress of Neurological Surgeons 2019.

This is an Open Access article distributed under the terms of the Creative

Commons Attribution-

NonCommercial-NoDerivs licence (http:// creativecommons.org/licenses/by-nc-nd/ 4.0/), which permits non-commercial reproduction and distribution of the work, in any medium, provided the original work is not altered or transformed in any way, and that the work is properly cited. For commercial re-use, please contact

journals.permissions@oup.com

\section{Single-Arm, Open-Label, Multicenter Study to Evaluate the Safety and Performance of Dura Sealant Patch in Reducing Cerebrospinal Fluid Leakage Following Elective Cranial Surgery: The ENCASE Trial Study Protocol}

BACKGROUND: Cerebrospinal fluid (CSF) leakage is one of the most common neurosurgical complications, occurring in $4 \%$ to $32 \%$ of surgical cases, with a higher incidence in complicated skull base surgery, intradural spine surgery, and the surgery of the posterior fossa. Our group developed a Dural Sealant Patch (DSP) for watertight dural closure after cranial surgery.

OBJECTIVE: To clinically study for the first time the safety and performance of the DSP as a means of reducing CSF leakage in patients undergoing elective cranial intradural surgery with a dural closure procedure.

METHODS: We will conduct an open-label, single-arm, multicenter study with a $360 \mathrm{~d}$ (12 mo) follow-up. A total of 40 patients will be enrolled at 3 sites. The primary endpoint is a combination of occurrences of one of the following events: postoperative percutaneous CSF leakage, intraoperative leakage at $20 \mathrm{~cm} \mathrm{H}_{2} \mathrm{O}$, or postoperative wound infection. The secondary endpoints are pseudomeningocele and thickness of dura + DSP.

EXPECTED OUTCOMES: Not more than 3 patients will meet the primary endpoint suggesting safety and efficacy.

DISCUSSION: As a next step, a randomized controlled trial against the best current practice will follow to evaluate if DSP reduces CSF leakage while its safety is noninferior.

KEY WORDS: Dura, Sealing, CSF, Leakage, Prevention

Neurosurgery 0:1-6, 2019 DOI:10.1093/neuros/nyz396 www.neurosurgery-online.com

\section{GENERAL INFORMATION}

Title: Evaluate the Safety and Performance of Dura Sealant Patch in Reducing CSF Leakage Following Elective Cranial Surgery

Study Dates: October 11, 2018 to December 2019

Funding Agency:

ABBREVIATIONS: AEs, adverse events; $\mathrm{Cl}$, confidence interval; CSF, cerebrospinal fluid; DSMB, Data Safety Monitoring Board; DSP, Dura Sealant Patch; e-CRF, electronic case report form; EDC, electronic data capturing; GCP, good clinical practice; ICF, Informed Consent Form; MDD, Medical Device Directive; MRI, magnetic resonance imaging; PEEP, positive end expiratory pressure; SAE, (serious) adverse event
Polyganics BV

Rozenburglaan 15A

9727 DL Groningen, The Netherlands

Registry: Clinicaltrials.gov, NCT03566602

Institutional Approvals:

Approved by the Dutch Medical Ethical Board (Utrecht, The Netherlands; number NL64477.041.18)

Approved by the Swiss Medical Ethical Board (Zurich, Switzerland; number BASEC 20180 1073)

\section{Investigators:}

Research site 1, principal investigator:

Dr T. P. C. van Doormaal, MD, PhD.

Rudolf Magnus Institute of Neuroscience

Department of Neurosurgery

University Medical Center Utrecht, G.03.124 
Heidelberglaan 100

3584 CX Utrecht, The Netherlands

Research site 2:

ETZ Hospital (responsible investigator: Dr P. R. A. M. Depauw)

Department of Neurosurgery

Hilvarenbeekseweg 60

5022 GC Tilburg, The Netherlands

Research site 3:

Universitatspital Zurich (responsible investigator: Dr M. R. Germans)

Department of Neurosurgery

Frauenklinikstrasse 10

8091 Zurich, Switzerland

\section{RATIONALE AND BACKGROUND INFORMATION}

Cerebrospinal fluid (CSF) leakage is one of the most common neurosurgical complications, occurring in $4 \%$ to $32 \%$ of surgical cases, with a higher incidence in complicated skull base surgery, intradural spine surgery, and the surgery of the posterior fossa. ${ }^{1,2}$ Most patients with CSF leakage necessitate a prolonged hospital stay, antibiotic treatment for meningitis, external lumbar drainage, reoperation, or a combination of these measures. CSF leakage leads to a significant patient burden and expense, with an estimated cost of 10000 to 15000 US dollars per patient per leakage. ${ }^{2}$ The use of a dural sealant as an adjunct to primary dural closure is often assumed to prevent further CSF leakage. However, until now no sealant has shown to have a significant reducing effect. ${ }^{3}$

The sponsor of this study (Polyganics BV, Groningen, The Netherlands) has developed in close cooperation with the Brain Technology Institute (Utrecht, The Netherlands) a Dural Sealant Patch (DSP) for watertight dural closure after cranial surgery. Preclinical studies showed a better adherence to dura and burst pressures for this sealant than currently used sealants. (Submitted) biological safety hazards of DSP have been addressed. Based on these data, DSP was considered safe for implantation. Until the current study, DSP was not tested in human subjects.

\section{STUDY GOALS AND OBJECTIVES}

The objective of this study is to clinically assess the safety and performance of DSP as a means of reducing intra- as well as postoperative CSF leakage in patients undergoing elective cranial intradural surgery with a dural closure procedure.

\section{Primary Endpoint}

A composite endpoint of any neurosurgical event is defined as follows.

\section{Safety}

Incidence of wound infection within $30 \mathrm{~d}$ defined in accordance with the Centers for Disease Control and Prevention guidelines for superficial incisional, deep incisional, and organ space infections.

\section{Performance}

Incidence of intraoperative CSF leakage after patch application at a positive end expiratory pressure (PEEP) of $20 \mathrm{~cm} \mathrm{H}_{2} \mathrm{O}$.

Incidence of percutaneous CSF leak confirmed by a $\beta-2$ transferrin test up to $30 \mathrm{~d}$ after surgery.

\section{Secondary Endpoints}

Safety

Incidence of device-related adverse events (AEs) throughout the study up to $90 \mathrm{~d}$ after surgery.

Incidence of device-related AEs throughout the study up to $360 \mathrm{~d}$ after surgery.

Incidence of wound infection confirmed by an increase in Creactive protein (CRP) and positive cultures up to $90 \mathrm{~d}$ after surgery.

\section{Performance}

Incidence of percutaneous CSF leakage confirmed by a $\beta$-2 transferrin test up to $90 \mathrm{~d}$ after surgery.

Incidence of pseudomeningocele with the need of puncture, external lumbar drainage, or surgical evacuation, as assessed by the treating physician, up to $90 \mathrm{~d}$ after surgery.

Incidence of pseudomeningocele $>20 \mathrm{cc}$, as confirmed on magnetic resonance imaging (MRI).

Thickness of dura mater and DSP (combined) in mm analyzed with MRI.

\section{Additional Endpoints}

Incidence of complications requiring a reintervention up to $30 \mathrm{~d}$ after surgery.

Ease of use and application of the DSP (standardized questionnaire).

\section{STUDY DESIGN}

This study will be conducted as an open-label, single-arm, multicenter study with a $360 \mathrm{~d}$ (12 mo) follow-up. In this study, each subject will receive 1 DSP after the closure of the dura mater. The dura mater will be closed with suturing. If deemed necessary by the surgeon, a substitute (autologous tissue only) can be used.

The assessments performed in this study as well as the timepoints are described in the study scheme (Figure 1).

Follow-up of the subjects will be performed on day 7 (or at discharge, whichever comes first) and at 30, 90, and $360 \mathrm{~d}$ after cranial surgery.

\section{Research Population}

This study is planned to enroll up to 40 subjects at 3 sites, scheduled for elective cranial surgery. We aim to stratify and 


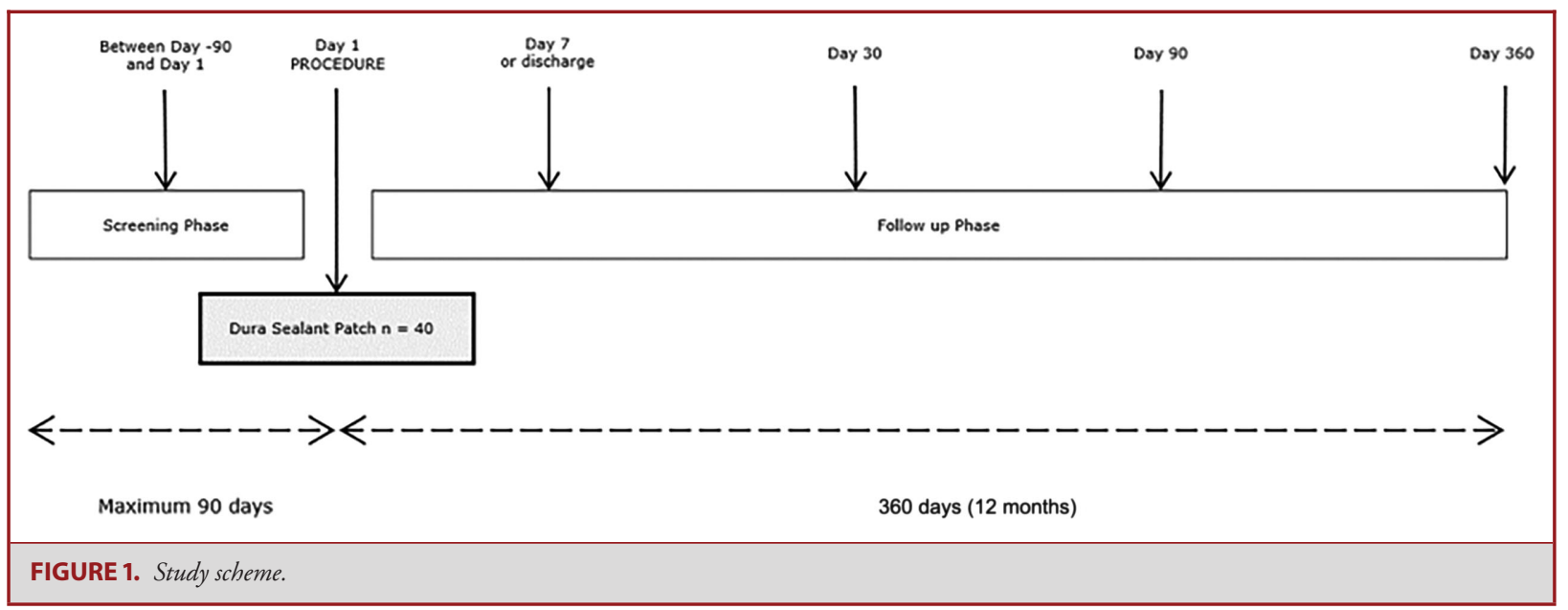

enroll 34 supra- and 6 infratentorial trepanations. A maximum of 25 subjects will be enrolled per site.

\section{Inclusion/Exclusion Criteria}

\section{Preoperative Inclusion Criteria}

- Subjects who are able to provide a written informed consent prior to participating in the clinical investigation.

- Subjects who are $\geq 18$ yr old.

- Subjects who are able to comply with the follow-up or other study requirements.

- Subjects who are planned for an elective intracranial intradural surgery in whom a dural incision of at least $2 \mathrm{~cm}$ in length is necessary, which will be closed.

- Female subjects of child-bearing potential must agree to use any form of contraception from the time of signing the informed consent form through $90 \mathrm{~d}$ postsurgery.

\section{Preoperative Exclusion Criteria}

- Female subjects who are pregnant or breastfeeding.

- Subjects with an assumed impaired coagulation due to medication or otherwise.

- Subjects suspected of an infection requiring antibiotics.

- Subjects with any type of dural diseases in the planned dural closure area.

- Subjects requiring reopening of the planned surgical area within $90 \mathrm{~d}$ after surgery.

- Subjects requiring local radiotherapy in the planned surgical area.

- Subjects with a known allergy to any of the components (lactide-caprolactone copolyester; butanediol-BDI copolyurethane; polyethylene glycol succinimidyl gluterate; disodium hydrogen phosphate or D\&C Green No 6) of the DSP.

- Subjects who previously participated in this study or any investigational drug or device study within $30 \mathrm{~d}$ of screening.

- Subjects with a presence of hydrocephalus.
- Subjects with a contraindication to MRI [cardiac pacemaker or defibrillator, severe claustrophobia, injured by a metallic object that was not removed, cochlear (ear) implants, metallic implants (eg, knee replacement)].

\section{Intraoperative Inclusion Criteria}

Subjects will be eligible according to the following criteria:

- Surgical wound classification Class I/Clean.

- Minimally $5 \mathrm{~mm}$ of dural space surrounding the dural opening.

\section{Intraoperative Exclusion Criteria}

- Subjects in whom the elevation of PEEP or $\mathrm{pCO}_{2}$ has a potential detrimental effect.

- Subjects who will require a CSF or wound drain, electrodes, or other devices passing the dural layer or extra to intracranial bypass surgery.

- Primary closure of the dura mater with synthetic, nonautologous or autologous material other than galea.

- A gap >3 mm after the primary closure of the dura mater.

- Dural opening cannot be covered by DSP $(8$ by $8 \mathrm{~cm}$ ) with a 5-mm overlap.

\section{Investigational Device Description}

The bioresorbable DSP is indicated for use as an adjunct to standard methods of dural closure, such as suturing, to provide a watertight closure of the dura mater to prevent CSF leakage after the dural closure procedure.

DSP is a flexible patch that consists of two layers: the adhesive layer (white) and the sealing layer (blue) (Figure 2). The white adhesive layer is foam-shaped and consists of bioresorbable copolyester. The white foam will strongly adhere to the dura due to the incorporated Polyethylene glycol-N-hydroxysuccinimide (PEG-NHS) adhesive component and buffer salt. This layer reacts with amines in the dural tissue in a moist environment, forming covalent bonds between the device and the tissue. 


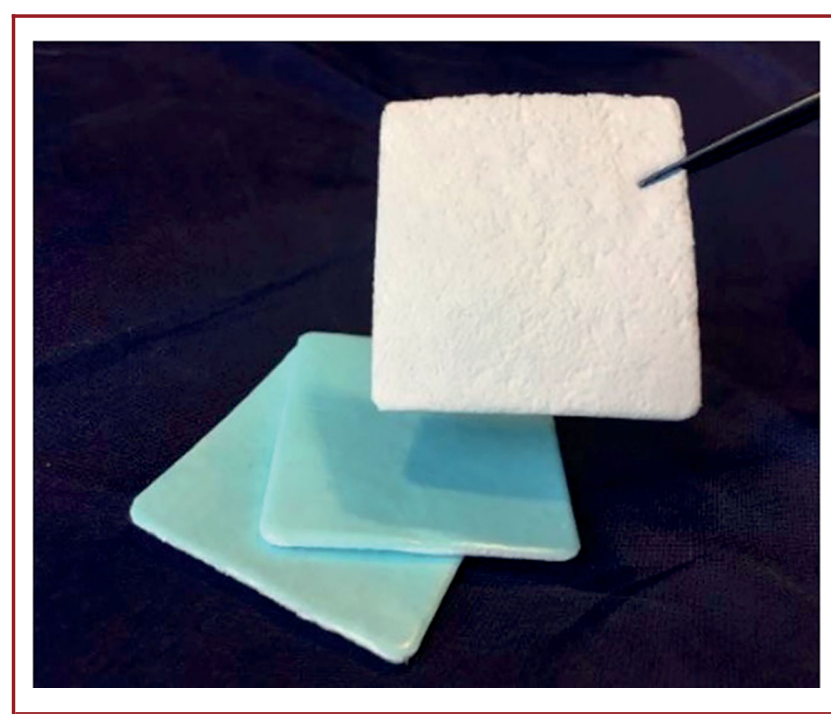

FIGURE 2. Investigational device. Length $8 \mathrm{~cm}$, width $8 \mathrm{~cm}$, and weight 1600 to $2000 \mathrm{mg}$.

The other side of the product, the watertight sealing layer, is made from blue-colored bioresorbable polyurethane (PU). The colorant is added to clearly distinguish between the sides of the product, so the correct (white) side will be placed to the dura.

\section{METHODOLOGY}

\section{Device Implantation}

- The package with DSP is taken out of the freezer at least 10 min and maximum of $8 \mathrm{~h}$ before use.

- Dura mater should be closed with the standard method of suturing.

- The dura mater surface is rinsed from particles (such as bone dust) with a physiological saline.

- Dura mater surface should be moist (remove excessive fluid if applicable).

- Hemostasis should be achieved.

- Open the aluminum pouch and also the inner blister (both not sterile).

- DSP is cut into the required size.

- The white side of the dry DSP is positioned against the sutured area of the dura mater if

$\circ$ maximum gap between sutures is $3 \mathrm{~mm}$ or smaller; and

- minimal cover is $5 \mathrm{~mm}$ beyond the margins of the gaps in the sutured area at all edges.

- A moist gauze is used to compress the DSP for $2 \mathrm{~min}$.

\section{Measurements}

\section{Preoperative}

Demographic information will be collected at screening to obtain information regarding gender, child-bearing potential, age, length, weight, and body mass index of the patient. Also, information will be collected about the subjects' medical as well as surgical history (ie, reason for surgery, allergies, tobacco use, use of medication). Comorbidity will be collected at screening as well.

\section{Intraoperative}

Surgery indication, used device size (original or adjusted by cutting), LOT number, location of incision, primary technique for the closure of the dura (with or without galea), and type and size of suture. The type of suture should be absorbable.

To determine the intraoperative CSF leakage before and after the application of the DSP, the CSF pressure will be raised by increasing PEEP and eventually, if needed, $\mathrm{pCO}_{2}$. The PEEP will be increased to $20 \mathrm{~cm} \mathrm{H}_{2} \mathrm{O}$ for $20 \mathrm{~s}$. In case the level of saline/CSF does not rise during this interval, the PEEP is returned to normal and the end-tidal $\mathrm{pCO}_{2}$ will be increased in acceptable steps to a maximum of $6.5 \%$ for $60 \mathrm{~s}$. In case the level of saline/CSF does not rise during this interval, this will be combined with a PEEP increase to $20 \mathrm{~cm} \mathrm{H}_{2} \mathrm{O}$ for $20 \mathrm{~s}$.

First, this test will be performed before the closure of the dura to determine the safety for the postoperative intracranial field (control of hemorrhage, swelling, or other potential adverse effects) and to determine at what point the CSF rises.

Upon completion of the primary sutured dural closure and before the application of the DSP, the closure of the dura will be evaluated for CSF leakage by repeating the test.

After the application of the DSP and before the closure of the cranium, the PEEP (and if applicable the $\mathrm{pCO}_{2}$ ) elevation test will be performed for the third time to evaluate CSF leakage.

A photograph will be taken before and after the device application. This photograph will need to include the subject ID as well as a ruler. The ruler will need to have a metric system with at least a mm-scale. The photographs need to be uploaded into the electronic case report form (e-CRF).

\section{Follow-up}

On day 7 or discharge (whichever comes first), day 30, day 90 , and day 360, a physical exam will be performed.

All subjects will undergo an MRI on day 7 or discharge (whichever comes first) and on day 90. An MRI on day 30 will only be performed if there are clinical signs of leakage or there is a subcutaneous fluid pocket at any time point in the 30-d postoperative period. An independent radiologist will analyze the MRIs of all subjects for the presence of extradural CSF, volume of extradural fluid pockets, and thickness of dura plus sealant.

If external leakage from wounds is visible, a $\beta-2$ transferrin test will be performed.

During the hospitalization, the subject will be monitored daily for clinical signs of infection. The surgical wound will be inspected daily starting $24 \mathrm{~h}$ after surgery. Data will be collected from $24 \mathrm{~h}$ after surgery and thereafter every $24 \mathrm{~h}$ until day 7 or discharge (whichever comes first). Blood analysis will be performed in the case of clinical signs of infection. 
(Serious) adverse events (SAEs) will be documented directly after the application of the investigational device till the last follow-up contact.

After the procedure, surgeons will be invited to complete several closed-end questions regarding their user experience with DSP.

\section{DISCUSSION}

This is a first in human trial combining the safety and efficacy of the DSP at one endpoint. This trial will give a first suggestion on safety and efficacy. However, as a next step a randomized controlled trial against the best current practice will have to follow to evaluate if DSP significantly reduces CSF leakage while its safety is noninferior.

\section{TRIAL STATUS}

\section{Recruiting}

\section{Safety Considerations}

Preclinical studies showed a better adherence to dura and increased burst pressures than currently used sealants. (Submitted) biological safety hazards of DSP have been addressed to conform to ISO 10993 (biological evaluation of medical devices $)^{4}$ in a series of in-Vitro and/or in-Vivo studies: Cytotoxicity, Sensitization, Irritation, Acute, Sub-Acute and Sub-Chronic toxicity, Pyrogenicity, Hemocompatibility, Genotoxicity, Neurotoxicity, Local Effects and In-Vivo Degradation up to 12 months (submitted). Based on these data, DSP was considered safe for implantation.

\section{DATA MANAGEMENT AND STATISTICAL ANALYSIS}

Research coordinators at the clinical site will perform primary data collection drawn from source document (hospital records) reviews. The e-CRF will be completed on a continuous basis starting from the point of enrolling the subject to final followup. The sponsor will provide clinical monitoring, including a review of e-CRF with verification against the source documentation. This may include worksheets retained with the e-CRF documentation and hospital records.

Data will be collected through an electronic data capturing (EDC) system on the e-CRF, a secure, internet-based case report form and image transfer software. This system will be used to record all subject information collected in the clinical investigation for secure data tracking and centralized data monitoring ("remote monitoring").

Automated, real-time data analyses built into the database enable complete control on study outcomes and safety assessments.

The principal investigator or his/her designee at the clinical site will perform primary data collection by entering the data into the e-CRF, using a standard internet browser. Only the principal investigator or other predesignated clinical investigation site personnel will be authorized to enter data (from source documents) via the internet-based e-CRF, using a unique user name and password. Each user access to the system will be tracked so that all data operations can be monitored and verified.

\section{Monitoring}

The sponsor's designated monitor shall ensure appropriate training is provided to all site personnel involved prior to the start of the clinical investigation.

The principal investigators, using their personal login information, shall approve and date each case report form section in the EDC system.

The monitor, using his/her personal login information, shall verify all critical data points against the source documents and issue electronic queries for the authorized clinical site personnel to respond.

A case report form section shall be considered complete when all data are entered, verified by the monitor, outstanding queries resolved, and signed off by the principal investigator.

A critical quality control shall be performed for the first 2 subjects by the sponsor's designated data management team and queries issued where needed.

After the monitor has done the source document verification and obtained satisfactory answers to eventual queries from the site, a full quality control shall be performed on the monitored data throughout the clinical investigation by the designated data management team and queries issued where needed. This process will be repeated till the end of the clinical investigation so as to allow for a timeline freezing of the database for statistical analysis.

\section{Statistical Analysis}

For the purpose of sample size calculation, the primary outcome measurement is assumed to be the composite of the three primary endpoints. Each patient will score "yes" if any of the three occurs and "no" otherwise. This binary outcome is assumed to follow a binomial distribution. The percentage of patients scoring "yes", along with an exact (Clopper-Pearson) two-sided 95\% CI for percentage, will be reported. Overall statistical success will be concluded if the percentage of patients scoring "yes" is 7\% or less. This is based on a previously reported neurological complications rate of $7.7 \% .^{5}$ As this is a single-group pilot study, the sample size calculation is based on specifying the width of the $\mathrm{CI}$ around the primary outcome measure, rather than aiming to demonstrate noninferiority or superiority to a given complication rate. If the percentage observed in the study were to be $7 \%, 35$ patients would be required for a CI with a width no more than $20 \%$. Allowing for $12.5 \%$ dropping out, the study will aim to recruit 40 patients.

\section{QUALITY ASSURANCE}

The study coordinator and investigators followed accredited good clinical practice (GCP) training and the study is performed 
according to GCP regulations. A Data Safety Monitoring Board (DSMB) will be installed. The DSMB will advise the sponsor regarding the continuing safety of current participants and those yet to be recruited. The DSMB will also review data relating to safety and performance and ensure the continued scientific validity and merit of the study. Interim safety analysis will be performed after 10 patients.

\section{EXPECTED OUTCOMES OF THE STUDY}

Not more than 3 patients will meet the primary endpoint suggesting safety and efficacy.

\section{DURATION OF THE PROJECT}

Inclusion-final 12 mo follow-up: 18 mo.

\section{PROJECT MANAGEMENT}

Principal Investigator: Dr T. P. C. van Doormaal, MD, PhD

Site Coordinator Zurich: Dr M. R. Germans, MD, PhD

Site Coordinator Tilburg: Dr P. R. A. M. Depauw, MD, PhD

Project Managers Sponsor: A. Piteira Banga, MSc, and B. J. Korteling, MSc

Clinical Research Manager Sponsor: E. Maas-Soer, MSc

\section{ETHICS}

\section{Regulation}

The study will be performed in accordance with the Medical Device Directive (MDD 93/42/EEC and MEDDEV 2.7/3 rev. 3; 2015), ${ }^{6}$ MEDDEV 2.7/4, ${ }^{7}$ World Medical Association Declaration of Helsinki, ${ }^{8}$ and ISO 14155:2011. ${ }^{\text {The ENCASE }}$ protocol was approved by the Medical Ethical Commission in Utrecht, the Netherlands (NL64477.041.18), the Dutch Inspection for Healthcare and Youth (IGJ), and the Swiss Medical Ethical Board (BASEC 2018-0 1073).

\section{Informed Consent}

Prior to signing the Informed Consent Form (ICF), patient information should be given in a language and fashion understandable to the patient. Information should be provided written as well as oral. Patients should not be coerced, persuaded, or unduly influenced to (continue to) participate in the study.
Patients should be given ample time (at least $24 \mathrm{~h}$ ) and opportunity to enquire information regarding the study, ask questions regarding their possible treatment and enrolment, and consider their participation. All questions should be answered to the satisfaction of the patient. Patient-informed consent must be obtained prior to any study activities. The ICF should be signed and dated by the patient, and the person conducting the informed-consent discussion. In case the patient is unable to read and understand the ICF, a witness should be present during the entire informedconsent discussion. This witness should also sign the ICF, stating that informed consent was freely given by the patient. The patient should receive one of the 2 signed and dated copies of the ICF.

\section{Disclosures}

This study is funded by Polyganics BV, Rozenburglaan 15A, 9727 DL Groningen, The Netherlands. Dr van Doormaal received a consultancy fee in the design phase of the product from Polyganics BV.

\section{REFERENCES}

1. Hutter G, von Felten S, Sailer MH, Schulz M, Mariani L. Risk factors for postoperative CSF leakage after elective craniotomy and the efficacy of fleece-bound tissue sealing against dural suturing alone: a randomized controlled trial. J Neurosurg. 2014;121(3):735-744.

2. Grotenhuis JA. Costs of postoperative cerebrospinal fluid leakage: 1-year, retrospective analysis of 412 consecutive nontrauma cases. Surg Neurol. 2005;64(6):490493, discussion 493-494.

3. Kinaci A, Algra A, Heuts S, O’Donnell D, van der Zwan A, van Doormaal T. Effectiveness of dural sealants in prevention of cerebrospinal fluid leakage after craniotomy: a systematic review. World Neurosurg. 2018;118:368-376.e1.

4. International Organization for Standardization. ISO 10993-1:2009: biologica evaluation of medical devices. International Organization for Standardization. https://www.iso.org/standard/68936.html. Accessed April 25, 2019.

5. Osbun JW, Ellenbogen RG, Chesnut RM, et al. A multicenter, single-blind, prospective randomized trial to evaluate the safety of a polyethylene glycol hydrogel (Duraseal Dural Sealant System) as a dural sealant in cranial surgery. World Neurosurg. 2012;78(5):498-504.

6. European Union. MEDDEV 2.7/3 REV 3: clinical investigations: serious adverse reporting under directives 90/385/EEC and 93/42/EC. European Union. http://ec.europa.eu/growth/sectors/medical-devices/current-directives/guidance_en. Accessed April 25, 2019

7. European Union. MEDDEV 2.7/4: guidelines on clinical investigation: a guide for manufacturers and notified bodies. European Union. http://ec.europa.eu/growth/sectors/medical-devices/current-directives/guidance_en. Accessed April 25, 2019.

8. World Medical Association. Declaration of Helsinki. https://www.wma.net/ policies-post/wma-declaration-of-helsinki-ethical-principles-for-medical-researchinvolving-human-subjects/. Accessed April 1, 2019.

9. International Organization for Standardization. ISO 14155:2011: clinical investigation of medical devices for human subjects - good clinical practice. International Organization for Standardization. https://www.iso.org/standard/45557.html. Accessed April 25, 2019. 